\title{
Two Sides to Every Story: Early Specialization in Medical Education
}

\author{
Sean T. Campbell ${ }^{1} \cdot$ Dattesh R. Dave $^{2}$
}

Published online: 18 October 2017

(C) International Association of Medical Science Educators 2018

\begin{abstract}
Early specialization has many benefits, including increased training efficiency, early career benefits, and decreased training costs. Additionally, external pressures such as the rising costs of medical education, work hour restrictions during training, and the expanding base of medical knowledge make continuous restructuring of medical training inevitable, and overall, beneficial. However, changes must be considered carefully to limit loss of breadth and training quality and ensure trainee knowledge of their field of choice.
\end{abstract}

Keywords Early specialization $\cdot$ Training quality $\cdot$ Medical education

\section{Introduction}

Training a physician requires an extensive investment of resources and ensuring competence is critically important. This is accomplished by strict guidelines regarding curriculum, length of training, and board certification [1]. However, medicine is evolving rapidly and training programs must adapt. The sheer volume of knowledge has led to increasing specialization, and work hour restrictions have been established, reducing the amount of training time available [2]. In some

Sean T. Campbell

campbellst87@gmail.com

1 Department of Orthopaedic Surgery, Stanford University, 300 Pasteur Drive, Room R144, Stanford, CA 94305-5341, USA

2 Department of General Surgery, University of California San Diego, San Diego, CA, USA countries, a shortage of primary care providers has been declared and costs of medical education have continued to rise $[3,4]$.

In response to these pressures, alterations to the traditional pathways to allow for earlier specialization have been proposed and created $[5,6]$. Examples of these include reduced duration of medical school, the combining of residency and fellowship programs to common "integrated" programs, among others. While there are a number of benefits to these programs, problems and concerns exist as well.

\section{In Favor of Early Specialization}

Early specialization has become an inevitability, with patients, trainees, and department chairs driving the demand. Here, we review the reasons in favor of early specialization with a focus on surgical training, including acceleration of career goals, diminished costs for the trainee, and the overall diminishing returns from extended general surgery training.

In terms of residency training, pursuing early specialization helps residency program directors recruit residents of higher academic caliber, results in earlier development of specialtyspecific technical prowess, and allows more time to pursue individual career goals. So-called integrated residency programs provide an excellent example; these are the training programs that combine what was previously taught in separate residency and fellowship programs into a single program, and the training is usually accomplished in a shorter period of time. In a survey of 92 plastic surgery program directors (PDs), over half reported that their integrated residents had stronger research experience than their fellows of the same specialty (who entered training at a later stage, plastic surgery fellowship after general surgery residency) [7]. Furthermore, $69.2 \%$ of PDs responded that higher academic achievement of 
entering residents might drive programs toward integrated models [7]. Previous studies have also demonstrated higher average board and in-service exam scores for integrated residents compared to traditional trainees [8]. Though the academic success of integrated residents has driven the transition toward the development of these programs, the technical success of integrated residents has helped maintain their popularity and demonstrate that decreased training time does not mean decreased quality of trainees.

While each institution's surgical caseload differs, a study of the Accreditation Council for Graduate Medical Education's (ACGME) case logging system demonstrated that integrated vascular residents performed more major, minor, and overall vascular cases than vascular surgery fellows (who began training in vascular surgery after general surgery residency) [9]. When factoring in cases vascular fellows performed during the 5 years of general surgery residency, their overall case volume only exceeded integrated vascular residents by an average of 50 cases [9]. Additionally, PDs of integrated residency programs have demonstrated confidence in the technical abilities of their graduates. In one study, $90 \%$ of "early specialization" program PDs reported that their graduating residents were ready for independent practice [8]. Most importantly to the resident, integrated programs offer a longer time period during which mentorship may be established, as well as a faster pathway to career advancement $[7,8]$.

Additionally, early specialization programs offer a pathway to counter the cost of rising medical education by decreasing the length of training. Integrated programs shorten overall surgical training in the USA from the typical 7-10 to 67 years. Between 1999 and 2009, the cost of undergraduate medical education in the USA has increased $21 \%$ for private schools and $80 \%$ for in-state residents of public medical schools [10]. Furthermore, post graduate debt can be substantial; fellowship-trained orthopedic surgeons averaged above $\$ 200,000$ of debt in one 2009 survey [10]. Fiscal analysis demonstrated that the average post fellowship orthopedist required an additional 9 years of practice for a positive return on investment of their fellowship [11]. While discussions of financial burden are necessary to consider, given the rising cost of medical education, ultimately, it is the changing nature of training that has driven the need for earlier specialization. Early specialization allows for reduced opportunity costs and early opportunities for physicians to begin practicing, earning income, and paying down debt.

It is enlightening to review the changes to general surgery residency training over the past few decades. General surgery training now includes formal rotations in trauma surgery, surgical critical care, minimally invasive surgery, colorectal surgery, surgical oncology, hepatobiliary surgery, and vascular surgery. Residents, therefore, are no longer trained by general surgeons but by multiple sub-specialists [12]. Each of these fields now demands fellowship training to achieve proficiency. Nearly $80 \%$ of general surgery residents plan to pursue fellowship, and recent American Board of Surgery surveys report that nearly $40 \%$ of graduating residents are not confident in their ability to practice independently [13, 14]. Fewer surgeons are practicing as general surgeons than even before, with a $25 \%$ decrease over the past two decades [8]. Furthermore, the proliferation of fellowships and integrated residencies has decreased surgical opportunities for general surgery residents [13]. This is most impactful in the third and fourth years of training - critical years to building competence for independent practice [15]. Given the depth each of the subspecialties demands, general surgery training has been diluted, thereby decreasing trainee confidence in performing all but the most basic procedures and driving the demand for specialization.

As early specialization programs become more popular, it is essential to consider the reasons driving these changes. Medical students are entering school at an older average age, graduating with more debt, and spending more time in training [16]. Furthermore, integrated surgical residents have demonstrated academic success, as well as the ability to enter independent practice after graduating. Ultimately, early specialization decreases training time and cost, increases patient access, and is beneficial to surgical training.

\section{Against Early Specialization}

Specialization has allowed physicians to remain up to date despite medicine's growing body of literature. Knowledge continues to grow but a physician must be an expert only with regard to a small percentage of all medical knowledge. Additionally, resources for training doctors are finite. In this setting, earlier specialization is inevitable and difficult to argue against completely. However, there are a number of drawbacks that should be considered: (1) loss of "breadth" in favor of "depth," (2) earlier trainee decision-making at a less mature stage, and (3) the potential for decreased residency training quality.

The discussion of breadth versus depth of knowledge is not unique to medicine. Breadth is described as "the full span of knowledge of a subject" and depth as "the extent to which specific topics are focused upon, amplified, and explored" [17]. It has been suggested that the most effective methods of education combine the acquisition of breadth and depth, so that students are first introduced to a broad overview for overall context and then later learn about specific facts [18]. This paradigm is applied in medical education: students are first introduced broadly to the biological sciences, then to a broad overview of medicine, and finally acquire significant depth of knowledge during specialty training. One potential problem with early specialization, then, is that trainees fail to 
obtain a broad enough education initially to place the later depth into appropriate context.

This concern is not new. As early as 1982, Gonnella and Veloski studied the effects of early specialization on US National Board Examination results among residents [19]. At that time, surgery, obstetrics and gynecology, pathology, and psychiatry training programs were moving toward early specialization. The authors found that trainees in more general programs had significantly higher scores on part III of the board exam, which is the final certifying exam for US physicians [19]. They concluded that in specialized programs, "knowledge and skills in the broad aspects of medicine are not adequately emphasized" [19]. Others have made arguments against early specialization in general surgery, describing early specialization and loss of breadth as partially responsible for physicians becoming "disease centered rather than patient centered," and for a system in which "medical care is by committee" [13]. One important drawback and concern is that physicians become so specialized in this model that they are no longer able to communicate effectively with members of other specialties or understand the broad picture that is important in the patient's care.

A second argument against early specialization is that important career decisions are made by younger trainees with less practical experience. With regard to medical students, New York University has created a three-year medical school curriculum, but students must choose their residency of choice at the time of admission [20]. Other accelerated pathways to fellowship require trainees (such as residents applying to fellowships that allow "skipping" the last year of residency training) to choose advanced subspecialization earlier and without full exposure to the field. Data has shown that medical students make different choices after direct exposure to a particular specialty [21]. It is likely that many trainees do not understand the full nature of the training they select, possibly demonstrated by physician surveys showing high rates of career dissatisfaction [22]. This ties into the concept of "burnout," which has been emphasized in the literature and affects half of American physicians [23]. It seems plausible that more complete exposure prior to career-altering decisions could improve trainee understanding of their future practice. This is one area of early specialization that is likely incompletely studied and the future effects are unknown.

Finally, there is concern that early specialization leads to decreased training quality. This is best stated in a 2003 article [13], which argues against proliferation of fellowships (advanced training following residency) in surgery. The author writes "...there is a price to pay for increased specialization in surgery. One is the cost to surgical residencies; to add more specialization because the average residency does not provide specific experience will certainly lower the quality of the superb residencies that do..." [13]. Because early specialization can lead to the creation and earlier migration of trainees into fellowship programs, it is one of the many contributors to the proliferation of fellowship training that is occurring. This leads to a diluted experience for residents and may obligate the pursuit of fellowship training to become competent, creating a repetitive and self-perpetuating cycle.

\section{Conclusion}

Early specialization has many benefits, including increased training efficiency, early career benefits, and decreased training costs. Additionally, external pressures and the expanding base of medical knowledge make continuous restructuring of medical training inevitable. However, changes must be considered carefully to limit loss of breadth and training quality.

\section{Compliance with Ethical Standards}

Conflict of Interest The authors declare that they have no conflict of interest.

\section{References}

1. Ten CO. Medical education in the Netherlands. Med Teach. 2007;29(8):752-7.

2. Meyers MO, Sarosi GA, Brasel KJ. Perspective of residency program directors on accreditation council for graduate medical education changes in resident work environment and duty hours. JAMA Surg. 2017. https://doi.org/10.1001/jamasurg.2017.2206.

3. Riley N, Withy K, Rogers K, DuBose-Morris R, Kurozawa T. Comparison of primary care physician reimbursement rates in the United States. Hawai'i J Med Public Health. 2017;76(3 Suppl 1):24.

4. Edeen R. The high price of a dream job: a four year look at the rising costs of medical education, funding, and the role of the medical student Americam Association of Medical Colleges. AAMC retreived June $28^{\text {th }}, 2017$. https://www.aamc.org/download/ 276596/data/thehighpriceofadreamjob.pdf.

5. Barondess JA. Specialization and the physician workforce: drivers and determinants. JAMA. 2000;284(10):1299-301.

6. Cassel CK, Reuben DB. Specialization, subspecialization, and subsubspecialization in internal medicine. N Engl J Med. 2011;364(12):1169-73.

7. Pace E, Mast B, Pierson JM, Leavitt A, Reintgen C. Evolving perceptions of the plastic surgery integrated residency training program. J Surg Educ. 2016;73(5):799-806.

8. Klingensmith ME, Potts JR, Merrill WH, Eberlein TJ, Rhodes RS, Ashley SW, et al. Surgical training and the early specialization program: analysis of a national program. J Am Coll Surg. 2016;222(4):410-6.

9. Pedersen RC, Lew WK, Patel K. Differences in case logs reports between early specialization and traditional vascular surgery training programs. J Vasc Surg. 2014;59(6):57S-8S.

10. Hwang JS, Beebe KS, Benevenia J, Karanfilian B, Berberian WS. Financial debt of orthopedic residents. Orthopedics. 2012;35(4): e549-54.

11. Gaskill T, Cook C, Nunley J, Mather RC. The financial impact of orthopaedic fellowship training. J Bone Joint Surg Am. 2009;91(7): 1814-21. 
12. Longo WE, Bauer S, Duffy A, Seashore J, Udelsman R. Early specialization in surgery: the new frontier. Yale J Biol Med. 2008;81(4):187-91.

13. Ferguson $\mathrm{C}$. The arguments against fellowship training and early specialization in general surgery. Arch Surg. 2003;138(8):915-6.

14. Flexible rotations during general surgery. American board of surgery website. Accessed June 2017. http://www.absurgery.org/ default.jsp?policyflexrotations.

15. Jensen AR, Nickel BL, Dolejs SC, Canal DF, Torbeck L, Choi JN. Impact of integrated programs on general surgery operative volume. Am J Surg. 2017;213(3):460-3.

16. Jauhar S. From all walks of life-nontraditional medical students and the future of medicine. N Engl J Med. 2008;359:224-7.

17. Breath and Depth of Learning 2017. SUNY Empire State College website. Accessed May 2017. https://www.esc.edu/degreeplanning-academic-review/evaluator-resources/assessing-learning/ breadth-and-depth/.
18. Hirsch EDJ. Seeking breadth and depth in the curriculum. Educ Leadersh. 2001;59(2):22-25.

19. Gonnella JS, Veloski JJ. The impact of early specialization on the clinical competence of residents. N Engl J Med. 1982;306(5):2757.

20. Accelerated Three-Year MD. NYU School of Medicine website. Accessed May 2017. https://med.nyu.edu/education/md-degree/ accelerated-three-year-md.

21. Branstetter BF, Faix LE, Humphrey AL, Schumann JB. Preclinical medical student training in radiology: the effect of early exposure. Am J Roentgenol. 2007;188(1):W9-W14.

22. Medscape Physician Compensation Report 2016. Medscape website. Accessed June 2017. http://www.medscape.com/features/ slideshow/compensation/2016/public/overview\#page $=17$.

23. Peckham C. Medscape Physician Compensation Report 2016. Slide 17. Medscape website. http://www.medscape.com/features/ slideshow/compensation/2016/public/overview\#page $=17$. Accessed June 2017. 